\title{
Intraoperative neurophysiological monitoring in spine surgery: indications, efficacy, and role of the preoperative checklist
}

\author{
Rishi R. Lall, M.D., ${ }^{1}$ Rohan R. Lall, M.D., ${ }^{1}$ Jason S. Hauptyan, M.D., Ph.D., ${ }^{2}$ \\ Carlos Munoz, B.A., ${ }^{1}$ George R. Cybulski, M.D., ${ }^{1}$ Tyler Koski, M.D., 1 \\ Aruna Ganju, M.D., Richard G. Fessler, M.D., Ph.D., ${ }^{1}$ and Zachary A. Smith, M.D. ${ }^{1}$ \\ ${ }^{1}$ Department of Neurological Surgery, Feinberg School of Medicine, Northwestern University, Chicago, \\ Illinois; and ${ }^{2}$ Department of Neurological Surgery, David Geffen School of Medicine, University of \\ California, Los Angeles, California
}

\begin{abstract}
Spine surgery carries an inherent risk of damage to critical neural structures. Intraoperative neurophysiological monitoring (IONM) is frequently used to improve the safety of spine surgery by providing real-time assessment of neural structures at risk. Evidence-based guidelines for safe and efficacious use of IONM are lacking and its use is largely driven by surgeon preference and medicolegal issues. Due to this lack of standardization, the preoperative sign-in serves as a critical opportunity for 3-way discussion between the neurosurgeon, anesthesiologist, and neuromonitoring team regarding the necessity for and goals of IONM in the ensuing case. This analysis contains a review of commonly used IONM modalities including somatosensory evoked potentials, motor evoked potentials, spontaneous or free-running electromyography, triggered electromyography, and combined multimodal IONM. For each modality the methodology, interpretation, and reported sensitivity and specificity for neurological injury are addressed. This is followed by a discussion of important IONM-related issues to include in the preoperative checklist, including anesthetic protocol, warning criteria for possible neurological injury, and consideration of what steps to take in response to a positive alarm. The authors conclude with a cost-effectiveness analysis of IONM, and offer recommendations for IONM use during various forms of spine surgery, including both complex spine and minimally invasive procedures, as well as lower-risk spinal operations.

(http://thejns.org/doi/abs/10.3171/2012.9.FOCUS12235)
\end{abstract}

KEY WORDS $\bullet \quad$ spine surgery $\bullet \quad$ intraoperative neurophysiological monitoring $\bullet$
preoperative checklist $\quad \bullet \quad$ somatosensory evoked potential $\bullet \quad$ motor evoked potential

$\mathrm{E}$ VEN in the most skilled hands, spine surgery carries an inherent risk of damage to critical neural structures with subsequent development of postoperative neurological deficits. To enhance the safety of spine surgery techniques, many surgeons use IONM. Although this technology cannot directly prevent intraoperative neurological injury, it has the potential to provide real-time feedback of critical neurological pathways to the surgeon. In select circumstances, this feedback may prevent or mitigate neurological injury. As evidenced by 1 large multicenter study, spinal operations for deformity correction that incorporate the feedback of an experienced neurophysiology team can have as much as a

\footnotetext{
Abbreviations used in this paper: $\mathrm{CMAP}=$ compound muscle action potential; EMG = electromyography; IONM = intraoperative neurophysiological monitoring; $\mathrm{MEP}=$ motor evoked potential; $\mathrm{SCI}$ $=$ spinal cord injury; SSEP = somatosensory evoked potential.
}

$50 \%$ lower rate of neurological deficits. ${ }^{46}$ That said, there is no established consensus regarding the use of IONM in routine and complex spine surgery. Given the current state of the health care economy and changes in reimbursement practices, this issue has become particularly relevant when considering the use of IONM in lower-risk spinal procedures. To date, the routine use of IONM in all spine surgeries remains controversial and varies between centers.

There are few high-quality studies regarding the particular indications for IONM in spine surgery, which has led to the lack of firm consensus or evidence-based guidelines for safe and efficacious implementation of neuromonitoring. ${ }^{19,22}$ Successful use of this technology requires fluid and coordinated 3-way communication between the neurosurgeon, anesthesiologist, and neurophysiologist.

The preoperative sign-in period serves as a critical time to integrate the separate goals of each member of the 
operative team in an effort to coordinate communication during the ensuing procedure. The preoperative sign-in period includes a discussion of the appropriate anesthetic protocol, the surgeon's goals and concerns, and what actions will be taken in the setting of a change in signal amplitude, latency, or threshold. ${ }^{50}$

At the authors' institution (Northwestern University), we have sought to incorporate a discussion of the role of IONM into the preoperative sign-in period. This discussion takes place prior to positioning of the patient and prior to IONM setup. The sign-in is always led by a surgeon and includes a discussion of the role of IONM in relation to the clinical goals of the ensuing case. Both the anesthesia and monitoring teams are expected to participate in this discussion and all questions are addressed. In particular, it is critical to discuss triggers for surgeon notification during the case. In this paper, we review modern techniques in neurophysiological monitoring, focusing on efficacy, limitations, and costs of utilization. Additionally, we review the available literature on operative checklist research and offer suggestions for incorporation of IONM into the preoperative sign-in period.

\section{Literature Search Criteria}

The terms "neurophysiological monitoring," "spine surgery," "somatosensory evoked potentials," "motor evoked potentials," "EMG," and "cost" were used as keywords to query the MEDLINE database. Both abstracts and full-text reports were reviewed, whereas case reports were excluded. Expert opinion was sought from academic spine surgeons specializing in minimally invasive techniques, deformity surgery, and intradural surgery, as well as neuroanesthesiologists and neuromonitoring specialists.

\section{Somatosensory Evoked Potentials}

Somatosensory evoked potentials are the most widely available and commonly used monitoring modality in spine surgery. ${ }^{38}$ Initially described by Nash et al. in $1977,{ }^{43}$ distal stimulating electrodes are placed on the limbs, and ascending sensory signals are recorded via scalp or posterior neck electrodes. The most common sites for stimulation are the posterior tibial and peroneal nerves for the lower extremities, and median and ulnar nerves for the upper extremities. ${ }^{39,40,46}$ Somatosensory evoked potentials directly monitor the dorsal column-medial lemniscus pathway. As such, SSEPs do not directly monitor corticospinal activity. Accordingly, SSEPs may provide false negatives in the setting of focal corticospinal tract injury as is noted in anterior spinal artery syndrome. There have been several reports of paraparesis following anterior SCI, with preserved SSEPs intraoperatively. ${ }^{4,21,34,42,53}$

Somatosensory evoked potentials are monitored continuously throughout surgery. Significant changes include amplitude decrease greater than $50 \%$ or increases in latency of more than $10 \%$ from baseline; these changes should be communicated to the surgeon. As a preliminary course of action, SSEP changes should prompt a search for correctable causes such as hypothermia, hypotension, use of halogenated anesthetics, and technical issues related to the IONM equipment. This trouble-shoot- ing requires timely and effective communication between the anesthesiologist, surgeon, and neuromonitoring staff. If this investigation is unrevealing, consideration should be given to reversing any recent surgical maneuvers, particularly those temporally associated with the monitoring change, and assessing for improvement in SSEP signal.

Nuwer et al. in $1995^{46}$ reported on results of a multicenter survey of members of the Scoliosis Research Society. This survey yielded 51,263 cases in which SSEPs were used as the sole mode of neuromonitoring, with a sensitivity of $92 \%$ and a specificity of $98 \%$ for new postoperative motor deficits. More recently, others have reported lower sensitivities ranging from $0 \%$ to $52 \%$, with specificities ranging from $95 \%$ to $100 \%$ (Table 1). ${ }^{23,26,29,51,54,58,59}$

A critical limitation of SSEP monitoring is the requirement for temporal summation. Readings are based on calculated averages and thus may take several minutes to change following an acute insult. In 2004, Hilibrand et al. ${ }^{26}$ compared cases monitored using both MEPs and SSEPs and found that SSEP changes lagged behind MEP changes by an average of 16 minutes. Thus, a major concern of SSEP monitoring is that by the time monitoring changes become apparent, irreversible neurological injury may have already occurred. Additionally, SSEPs have a low sensitivity for detection of nerve root injury and thus may miss damage caused by aberrant pedicle screw placement or nerve root traction. These deficiencies limit the overall efficacy of SSEPs (Table 2) as a standalone monitoring tool; thus, SSEPs should be used primarily as an adjunct to other forms of monitoring.

\section{Motor Evoked Potentials}

Direct monitoring of the corticospinal pathway became possible with the work of Merton and Morton in $1980,{ }^{41}$ who described transcranial magnetic stimulation of the motor cortex. This work allowed the development of transcranial MEP monitoring during spinal surgery with responses recorded either electromyographically via CMAPs or via epidural electrodes placed caudal to the region at risk (D-waves). Initially, transcranial MEPs had limited utility due to extreme sensitivity to volatile anesthetics. Work by Taniguchi et al. in $1993^{61}$ led to use of high-frequency $(>200 \mathrm{~Hz})$ multipulse electrical stimulation at relatively low voltages, which showed improved reproducibility and anesthetic resistance relative to earlier techniques. ${ }^{62}$ These improvements, in conjunction with improvements in intravenous anesthetic protocols, led to the widespread use of transcranial MEPs for monitoring of motor pathways during spine surgery. Compound muscle action potential and D-wave recording will be discussed separately below.

\section{Muscle MEPs}

Electromyographic recording of transcranial MEPs with CMAPs (muscle MEPs) allows for assessment of the entire motor axis including motor cortex, corticospinal tract, nerve root, and peripheral nerve. Recordings are usually performed at multiple upper- and lower-extremity muscle groups. Langeloo et al. ${ }^{31}$ found a sensitivity of $100 \%$ when monitoring at 6 sites in a series of 145 
Neurophysiological monitoring and spine surgery

TABLE 1: Significant studies reporting sensitivity and specificity of modern IONM techniques*

\begin{tabular}{|c|c|c|c|c|c|c|c|}
\hline Authors \& Year & $\begin{array}{l}\text { Type of } \\
\text { Monitoring }\end{array}$ & $\begin{array}{l}\text { No. of } \\
\text { Cases }\end{array}$ & Types of Cases & $\begin{array}{l}\text { Sensitivity } \\
(\%)\end{array}$ & $\begin{array}{l}\text { Specificity } \\
(\%)\end{array}$ & Warning Criterion & Modalities Used \\
\hline Nuwer et al., 1995 & SSEP & 51,263 & scoliosis & 92 & 98 & NA & NA \\
\hline Hilibrand et al., 2004 & SSEP & 427 & cervical spine (mixed) & 25 & 100 & NA & NA \\
\hline $\begin{array}{l}\text { Gunnarsson et al., } \\
2004\end{array}$ & SSEP & 213 & $\begin{array}{l}\text { thoracolumbar de- } \\
\text { formity }\end{array}$ & 29 & 95 & NA & NA \\
\hline Schwartz et al., 2007 & SSEP & 1,121 & scoliosis & 43 & 100 & NA & NA \\
\hline Smith et al., 2007 & SSEP & 577 & ACDF & 0 & 99 & NA & NA \\
\hline Kelleher et al., 2008 & SSEP & 1,055 & cervical spine (mixed) & 52 & 100 & NA & NA \\
\hline Park et al., 2011 & SSEP & 29 & kyphosis & 25 & 96 & NA & NA \\
\hline Calancie et al., 1998 & transcranial MEP & 32 & scoliosis & 100 & 100 & $\uparrow$ threshold $>100 \mathrm{~V}$ & NA \\
\hline Langeloo et al., 2003 & transcranial MEP & 145 & scoliosis + kyphosis & 100 & 91 & $\downarrow$ amplitude $>80 \%$ & NA \\
\hline Hilibrand et al., 2004 & transcranial MEP & 427 & cervical spine & 100 & 100 & $\downarrow$ lamplitude >60\% & NA \\
\hline Schwartz et al., 2007 & transcranial MEP & 1,121 & scoliosis & 100 & 100 & $\downarrow$ amplitude $>65 \%$ & NA \\
\hline Hsu et al., 2008 & transcranial MEP & 172 & scoliosis & 100 & 97 & $\downarrow$ amplitude >50\% & NA \\
\hline Kelleher et al., 2008 & transcranial MEP & 1,055 & cervical spine & 100 & 96 & not reported & NA \\
\hline Park et al., 2011 & transcranial MEP & 29 & kyphosis & 75 & 84 & all or nothing & NA \\
\hline Noonan et al., 2002 & multimodality & 134 & cervical spine & 100 & 95 & NA & $\begin{array}{l}\text { SSEPs \& neurogenic } \\
\text { MEPS }\end{array}$ \\
\hline Hilibrand et al., 2004 & multimodality & 427 & cervical spine & 100 & 100 & NA & $\begin{array}{l}\text { SSEPs \& transcranial } \\
\text { MEPs }\end{array}$ \\
\hline Schwartz et al., 2007 & multimodality & 1,121 & scoliosis & 100 & 100 & NA & $\begin{array}{l}\text { SSEPs \& transcranial } \\
\text { MEPs }\end{array}$ \\
\hline Quraishi et al., 2009 & multimodality & 102 & scoliosis + kyphosis & 100 & 84 & NA & $\begin{array}{l}\text { SSEPs \& either transcra- } \\
\text { nial MEPs or sponta- } \\
\text { neous EMG }\end{array}$ \\
\hline \multirow[t]{3}{*}{ Hamilton et al., 2011} & multimodality & $108,419 \dagger$ & all forms & 43 & 98 & NA & $\begin{array}{l}\text { for spinal cord deficit: } \\
\text { SSEPs + transcranial } \\
\text { MEPs }\end{array}$ \\
\hline & & & & 13 & 99 & NA & $\begin{array}{l}\text { for nerve root deficits: } \\
\text { SSEPs + EMGs }\end{array}$ \\
\hline & & & & 29 & 99 & NA & $\begin{array}{l}\text { for cauda equina deficit: } \\
\text { SSEPs + transcranial } \\
\text { MEPs }\end{array}$ \\
\hline
\end{tabular}

* ACDF = anterior cervical discectomy and fusion; NA = not applicable.

$\dagger$ Hamilton et al. report that $65 \%$ of reported cases used some form of neuromonitoring, but do not report what percentage used multimodality IONM.

consecutive patients, compared with a sensitivity of $88 \%$ when monitoring at only 2 sites. Because transcranial stimulation will induce movement, muscle MEPs preclude the use of neuromuscular blockade during surgery. It is absolutely essential to discuss this anesthetic concern preoperatively with the anesthesia providers. Additionally, muscle MEPs are only assessed periodically, in contrast to SSEPs that are assessed continuously throughout surgery. This represents a critical weakness of muscle MEPs and may lead to delayed recognition of neurological injury. Again, during the review of the preoperative checklist, a protocol for checking MEPs must be relayed to the neurophysiology team. This protocol ranges from only running MEPs at the surgeon's request, to periodic monitoring every set period of time.

A variety of different warning criteria are currently employed for interpretation of CMAP recordings. Ini- tially, most authors described an all-or-none criterion by which only a complete loss of CMAPs is considered clinically significant. More recently, Langeloo et al ${ }^{31}$ found that using a modified criterion of $80 \%$ decrease in amplitude at any single recording site yielded a sensitivity of $100 \%$; however, they reported 10 false positives using this criterion and a specificity of only $91 \%$. Calancie et al. in $1998^{8}$ reported a novel method of transcranial MEP interpretation, independent of amplitude. They assessed the minimum threshold stimulus required to generate distal CMAPs and found that an increase in threshold of more than $100 \mathrm{~V}$ yielded a sensitivity and specificity of $100 \%$ for postoperative motor deficits. Each of these criteria is considered valid, and the specific methodology used in any given case should be clarified preoperatively between the neurosurgeon and electrophysiologist.

Muscle MEPs are considered the gold standard for 
TABLE 2: Summary of strengths and weakness of modern IONM techniques

\begin{tabular}{|c|c|c|}
\hline Type of Monitoring & Strengths & Weaknesses \\
\hline \multicolumn{3}{|l|}{ SSEP } \\
\hline & broadly available \& relatively affordable & $\begin{array}{l}\text { averaging of evoked responses leads to significant delays in } \\
\text { signal change; may lag up to } 16 \text { mins behind transcranial MEP }\end{array}$ \\
\hline & allows continuous monitoring throughout case & does not directly monitor corticospinal tract \\
\hline & excellent specificity (approaching 100\%) & low sensitivity for motor deficit \\
\hline & may be used w/ neuromuscular blockade & may remain unchanged $w /$ anterior spinal artery injury \\
\hline & $\begin{array}{l}\text { warning criteria firmly established; decreased amplitude }>50 \% \\
\text { or increased latency }>10 \% \text { considered significant }\end{array}$ & \\
\hline \multicolumn{3}{|l|}{ transcranial MEP } \\
\hline & $\begin{array}{l}\text { excellent sensitivity for motor deficit, approaching } 100 \% \\
\text { directly evaluates entire motor axis (cortex, corticospinal tract, } \\
\text { nerve root, peripheral nerve) }\end{array}$ & $\begin{array}{l}\text { does not allow for continuous monitoring } \\
\text { precludes use of neuromuscular blockade \& causes patient } \\
\text { movement }\end{array}$ \\
\hline & $\begin{array}{l}\text { allows immediate assessment of corticospinal integrity following } \\
\text { high-risk maneuvers }\end{array}$ & highly sensitive to anesthetic effects \\
\hline & & $\begin{array}{l}\text { theoretical risk of inducing seizures, although no cases have } \\
\text { been reported }\end{array}$ \\
\hline \multicolumn{3}{|l|}{ D-wave } \\
\hline & $\begin{array}{l}\text { correlates most accurately w/ long-term motor function follow- } \\
\text { ing intramedullary spinal cord tumor resection }\end{array}$ & inaccurate in scoliosis surgery ( $27 \%$ false-positive rate) \\
\hline & $\begin{array}{l}\text { can be recorded continuously \& used w/ neuromuscular block- } \\
\text { ade }\end{array}$ & does not assess nerve root function \\
\hline & & $\begin{array}{l}\text { epidural recording electrode must be placed over spinal cord, \& } \\
\text { thus D-waves can only be used above T10-11 }\end{array}$ \\
\hline \multicolumn{3}{|l|}{ spontaneous EMG } \\
\hline & highly sensitive for nerve root injury & high rate of false positives \\
\hline & provides constant feedback throughout case & $\begin{array}{l}\text { extremely sensitive to temperature changes (cold irrigation or } \\
\text { use of cautery) }\end{array}$ \\
\hline & may be combined w/ SSEPs to improve specificity & precludes use of neuromuscular blockade \\
\hline \multicolumn{3}{|l|}{ triggered EMG } \\
\hline & high sensitivity for medial pedicle breach & optimal alarm criteria not firmly established \\
\hline & $\begin{array}{l}\text { useful in minimally invasive surgery where anatomical landmarks } \\
\text { may be challenging to visualize }\end{array}$ & $\begin{array}{l}\text { may provide false-positive alarms if multiple passes have been } \\
\text { made through pedicle or if operative field is bloody }\end{array}$ \\
\hline & relatively easy to perform \& interpret & $\begin{array}{l}\text { does not directly assess for neurological injury, only provides } \\
\text { information regarding pedicle integrity }\end{array}$ \\
\hline
\end{tabular}

detection of new postoperative motor deficits, with reported sensitivities ranging from $75 \%$ to $100 \%$ and specificities ranging from $84 \%$ to $100 \%$ (Table 1). ${ }^{8,26,27,51,58} \mathrm{Ad}$ ditionally, CMAP monitoring has been shown to provide earlier detection of spinal cord ischemia compared with SSEPs and D-waves, which may allow timely reversal of injury. ${ }^{36}$ Limitations of transcranial MEPs include sensitivity to volatile anesthetics as well; moreover, they cannot be continuously monitored throughout surgery. In addition, there is a theoretical risk of seizure induction secondary to transcranial stimulation, but there have been no reported cases of this induction in the literature (Table 2).

\section{Direct Waves (D-Waves)}

D-waves are generated via transcranial stimulation and monitored directly at the spinal cord level via placement of an epidural recording electrode caudal to the re- gion at risk. In contrast to CMAP monitoring, D-waves are relatively resistant to anesthetic effects and permit the use of neuromuscular blockade for paralysis (Table 2). ${ }^{13,57,65}$ In general, a $20 \%$ decrease in D-wave amplitude is considered to be a preliminary warning, whereas a $50 \%$ reduction in amplitude is considered indicative of significant neurological injury.

D-waves are infrequently used during deformity surgery due to a reported $27 \%$ false-positive rate, which Ulkatan et al..$^{65}$ propose is due to rotation of the corticospinal tract relative to the recording electrode during spinal curvature correction. Additionally, the lack of nerve root and cauda equina monitoring with D-waves may limit their utility in these cases. Instead, the predominant clinical application of D-waves lies in intramedullary spinal cord tumor resection. Kothbauer et al..$^{30}$ initially reported in 1998 that D-waves were superior to CMAPs in prediction of long-term motor status following intramedullary 
spinal cord tumor resection. Specifically, they found that patients with loss of CMAPs but preserved D-waves tended to have transient postoperative weakness that resolved by 1-2 month follow-up. In contrast, patients with loss of CMAPS as well as D-wave decrements greater than $50 \%$ were likely to have lasting motor deficit. Thus, use of Dwaves allowed for safer, more aggressive resection than would otherwise have been possible. These findings were subsequently reproduced by Sala et al.,${ }^{57}$ and D-waves are currently believed to be the gold standard for motor pathway monitoring during intramedullary spinal cord tumor resection. ${ }^{13}$

\section{Neurogenic MEPs}

Neurogenic MEPs were originally developed in the early 1990 s as an alternative means of motor monitoring that would bypass the complicated anesthetic requirements of transcranial MEPs. Neurogenic MEPs are generated via an epidural stimulating electrode placed rostral to the area at risk. Recordings are performed either at peripheral nerves or via an epidural recording electrode placed caudal to the region at risk. However, they have subsequently become controversial due to studies questioning whether neurogenic MEPs are truly monitoring motor signals. ${ }^{48,49,63,67}$ Initially, neurogenic MEPs were believed to represent descending motor activity, but Toleikis et al. in $2000^{63}$ performed collision studies suggesting that they instead represented antidromic dorsal column signal. These findings correlated with a report by Minahan et al. ${ }^{42}$ of 2 cases of postoperative paraparesis and normal sensory examination with preserved SSEPs and neurogenic MEPs intraoperatively. As such, current consensus is against the use of neurogenic MEPs as the sole method of motor pathway monitoring. ${ }^{13,22,39}$

\section{Spontaneous EMG}

Spontaneous or free-running EMG is widely used as a means of monitoring selective nerve root function during spinal cord surgery. During spinal cord instrumentation and pedicle screw placement, postoperative radiculopathy is more likely than SCI, making spontaneous EMGs optimal for these procedures. ${ }^{24}$ No stimulation is required for this technique, and continuous recordings are made from preselected muscle groups based on the nerve roots at risk. One muscle group per nerve root is generally considered adequate, but due to the high risk of C-5 palsy during cervical spine surgery, many surgeons prefer monitoring 2 muscle groups at this level, namely deltoid and biceps. ${ }^{5,18,28}$ At baseline, a healthy nerve root should have no muscle activity, that is, either a flat line or silence if audio feedback is equipped. During surgery, irritation of the nerve root due to traction or thermal injury will result in spikes or bursts of activity termed neurotonic discharges. With increasing degree of mechanical injury, amplitude and frequency of these discharges will increase and trains of activity may be observed. ${ }^{5,23,47}$ As with other forms of EMG monitoring, neuromuscular blockade is prohibited. In contrast to transcranial MEPs in which motor function is only assessed periodically, spontaneous EMG allows continuous feedback throughout the entire procedure.
Spontaneous EMG recordings are sensitive to temperature changes, and common causes of false spontaneous EMG activation include irrigation with cold water, and use of cautery devices. Gunnarson et al. ${ }^{23}$ reported spontaneous EMG activation at least once in $77.5 \%$ of 213 consecutive lumbosacral cases, which resulted in a sensitivity of $100 \%$, but a specificity of only $23.5 \%$. These results correlate with other studies of spontaneous EMG reporting high sensitivity and low specificity. ${ }^{5,28,54}$ The low specificity, however, may reflect the fact that spontaneous EMG is providing constant feedback to the surgeon, leading to alterations in surgical technique that may prevent a new neurological deficit and thus deceptively elevating the rate of false positives.

\section{Triggered EMG}

Triggered EMG was initially described by Calancie et al. in a porcine model in $1992^{9}$ as a means of assessing accuracy of pedicle screw placement. Triggered EMG relies on the concept that intact cortical bone should electrically insulate a well-placed pedicle screw from the adjacent nerve root. In contrast, with a medial pedicle breach, the pedicle screw would be relatively poorly insulated. Thus, by electrically stimulating the pedicle screw directly, and electromyographically assessing the lowest threshold voltage at which CMAPs are generated, one can assess the likelihood of medial pedicle breach. With the increasing prevalence of spinal instrumentation and fusion, triggered EMG has emerged as a popular means of preventing neurological injury in these cases. ${ }^{33,52,55,56}$ Triggered EMG has particular utility in minimally invasive spine surgery, in which visualization of anatomical landmarks is limited. In such cases, stimulation of pedicle taps and K-wires may be used to evaluate for accurate screw trajectory. Notably, in the setting of preoperative nerve root deficit, nerve conduction may be impaired, requiring higher thresholds for stimulation. Nerve conduction can be assessed intraoperatively by direct stimulation of the nerve root at low voltages and assessing for generation of CMAPs.

Several major studies have attempted to establish warning criteria for pedicle perforation in the lumbar spine, but consensus has yet to be reached. In 2007, Raynor et al. ${ }^{55}$ reported results of more than 4800 consecutive lumbar pedicle screw placements, with triggered EMG results compared with postoperative CT scans in each case. The authors found that with a threshold of more than $8.0 \mathrm{~mA}$, there was a $99.5 \%-99.8 \%$ likelihood of intraosseous screw placement $(95 \% \mathrm{CI})$, but the high false-positive rate at this threshold may lead to unnecessary delays in surgical time and revision of adequately placed screws. More recently, Parker et al..$^{52}$ reported results for 2450 consecutive lumbar screw placements and found that by using a threshold cutoff of less than $5 \mathrm{~mA}$, they were able to maintain an acceptable sensitivity of $43.4 \%$ for medial screw breech, while limiting the number of false positives.

Fewer studies have been performed to evaluate triggered EMG efficacy for thoracic pedicle screws. Raynor et al. ${ }^{56}$ reported using rectus abdominis CMAP record- 
ings for 677 consecutive thoracic pedicle screw placements. These authors found that above a threshold of 6.0 $\mathrm{mA}, 100 \%$ of screws were intraosseous. For screws with a threshold below $6.0 \mathrm{~mA}, 6(28.5 \%)$ of 21 screws were found to be medial breaches. Thus, the authors recommended using a threshold of less than $6.0 \mathrm{~mA}$ as a warning criterion for likely pedicle perforation.

The primary limitation of triggered EMG lies in the high rate of false-positive alarms. Common causes of false-positive monitoring include multiple passes within the same pedicle resulting in diminished pedicle integrity, and a wet field that may result in direct current conduction to the adjacent nerve root. Of note, most major studies on triggered EMG simply report rates of pedicle perforation, and there is a paucity of data on clinical correlation with these findings. Further studies are required to delineate the efficacy of triggered EMG in predicting true neurological compromise. Nonetheless, triggered EMG is an invaluable tool for improving safety in both minimally invasive and complex spine cases.

\section{Combined Multimodality IONM}

Multimodality monitoring has the potential to compensate for limitations of each individual monitoring modality and has become standard practice for a variety of spinal procedures. A combination of SSEP and MEP monitoring has long been used in scoliosis surgery for combined monitoring of ascending and descending pathways. The addition of spontaneous EMG and triggered EMG can enhance detection of nerve root injuries. Several studies have reported combined sensitivities and specificities approaching 100\% for combined multimodal neuromonitoring (Table 1). ${ }^{16,26,54}$ More recently, Hamilton et al. in $2011^{24}$ reported on rates of new neurological deficits in more than 100,000 patients operated on by members of the Scoliosis Research Society. For cases using multimodal neuromonitoring (mostly a combination of SSEPs with transcranial MEPs or EMG), sensitivity was reported as $43 \%$ for detection of new spinal cord deficit, $13 \%$ for new nerve root deficit, and $29 \%$ for new cauda equina deficit. These relatively poor results were in stark contrast to previously published reports and have generated significant debate on the sources of this discrepancy. ${ }^{17,45}$ This discussion is mostly speculative due to the nonspecific nature of the Scoliosis Research Society survey and the lack of data on IONM methodology in their study.

Several explanations have been proposed. First, the study does not specify how cases were classified in which a positive alarm led to a change in surgical approach. It is possible that many of these cases were actually true positives in which monitoring prevented a postoperative deficit, but they may have been incorrectly labeled as false positives due to the absence of postoperative deficit. Second, it is unclear whether a neurophysiologist team was present in all reported cases, and some have hypothesized that either surgeon-directed monitoring or automated monitoring may have led to the low sensitivities. ${ }^{17,45} \mathrm{Fi}$ nally, the lack of standardization in warning criteria for a positive alarm may have led to inconsistencies in data across multiple institutions. Nonetheless, this study high- lights the need for future prospective studies on the efficacy of multimodality neuromonitoring.

\section{Operative Checklists and Checklist Science}

Although the scientific literature on preoperative checklists remains incomplete, several studies to date have addressed the value of surgical checklists. In 2009, Haynes et al. ${ }^{25}$ studied the incorporation of the WHO's surgical safety checklist at 8 international hospitals and found a $30 \%$ reduction in surgical complications relative to historical rates. Although these findings make a strong argument for the use preoperative checklists, critics have questioned the generalizability of these results to higher-income countries with better-developed health care infrastructure. More recently, Calland et al. ${ }^{10}$ assessed the efficacy of surgical checklists by randomizing 47 laparoscopic cholecystectomies to either use or not use the preoperative checklist. No significant difference was found in patient outcomes or case times between the 2 groups, but the authors reported a decrease in subjective participant-reported levels of comfort, communication, and team efficiency.

In perhaps the most ambitious study to date on surgical checklists, Ziewacz et al. ${ }^{68}$ developed a pilot checklist for management of operating room crises. The authors identified 12 frequent operative crises including massive hemorrhage, air embolus, and ventricular tachycardia/fibrillation, and developed evidence-based protocols that they incorporated into a series of comprehensive checklists. Participants completed 8 simulation scenarios, 4 with the use of a checklist and preoperative education on how to properly use the checklist, and 4 without the checklist. The authors found a 6 -fold decrease in major deviation from evidence-based management guidelines in the group that used the crisis checklists. Although these results have yet to be correlated with a live clinical scenario, they offer a glimpse of the potentially pivotal role of operative checklists. These findings may be the most generalizable to neuromonitoring in spine surgery; development of a specific protocol to address changes in neuromonitoring signal may lead to a more systematic and standardized approach to these scenarios.

One critical barrier to checklist implementation is the persuasion of the surgical team and the operating room staff to commit to the process. Conley et al..$^{12}$ studied the process of implementing a surgical checklist in 5 Washington state hospitals and found that failure to persuasively explain why and how to use the checklist led to increased levels of frustration, and in some cases, complete abandonment of the protocol. This study highlights the critical role that surgeons, as leaders in the operating room, may play in encouraging a commitment to checklist implementation.

\section{Neuromonitoring and the Preoperative Sign-In}

As discussed above, the lack of standardization in neuromonitoring practices may contribute to inconsistency in the effectiveness and utility of neuromonitoring during spine surgery. As such, the preoperative discussion 
is a critical juncture for clarification of the goals and technique of monitoring in the case at hand. To this extent, we present a list of fundamental questions to be addressed by the neurosurgical, anesthesia, and electrophysiology teams prior to surgery. A summary of these questions can be found in Table 3 .

At the authors' institution, we have attempted to incorporate a discussion of neuromonitoring into the preoperative sign-in. This discussion takes place prior to positioning of the patient and setup of IONM equipment. It is institutional policy that an attending surgeon be present for the sign-in for all nonemergency cases. In an emergency setting, it is the responsibility of the attending surgeon to ensure that a resident with an appropriate level of training and experience is available. The surgical team leads the preoperative sign-in in all circumstances. Critical points for discussion include the goals of the surgery, special equipment required for the procedure, high-risk portions of the case, and potential complications. Although the degree of formality of the process is dependent on the specific team involved, it is the surgeon's responsibility to ensure that all critical issues are discussed and that all team members remain engaged in the process.

With regard to neuromonitoring, the discussion should initially focus on what forms of neuromonitoring are indicated in the case at hand. This discussion should be driven by the perceived likelihood of neurological injury as well as identification of anatomical structures at highest risk. In cases in which spinal cord deficits are most likely, SSEPs and transcranial MEPs are most likely to be indicated. If a posterior approach is being used, SSEPs may be sufficient, but anterior approaches most likely warrant transcranial MEPs due to the risk of anterior spinal artery syndrome. In cases in which nerve root deficits are of concern, spontaneous EMG and triggered EMG monitoring may be of value.

After selection of appropriate monitoring modalities, anesthetic requirements should be discussed. If transcranial MEPs are being used, halogenated anesthetics are contraindicated, and total intravenous anesthesia generally results in optimization of signal acquisition. Use of any form of EMG monitoring precludes the use of neuromuscular blockade. If paralytics are used, monitoring should be performed only after adequate reversal, that is, 3 or more twitches on train-of- 4 testing. ${ }^{39,47,55,56}$

Establishment of appropriate warning criteria is critical. For SSEP monitoring, a decrease in amplitude of more than $50 \%$ or an increase in latency of more than

TABLE 3: Summary of important IONM-related questions to include in the preoperative checklist

1. What monitoring modalities are most appropriate for the case at hand? What types of neurological deficits are most likely?

2. What anesthesia protocol will optimize acquisition of neuromonitoring signals? Is total intravenous anesthesia indicated? Can paralytics be used?

3. What alarm criteria will be used for each monitoring modality?

4. What actions will be taken in the setting of a positive alarm?

5. Are new techniques involved? How will they be implemented?
$10 \%$ is widely considered to be optimal. For other modalities such as muscle MEPs and triggered EMG, however, consensus has yet to be reached. Lower requirements for positive alarm criteria may improve sensitivity, but this must be balanced against the increased rate of false positives, which may contribute to unnecessary delays and changes in surgical plan. At the author's institution, for muscle MEPs, we consider a decrease in CMAP amplitude of more than $50 \%$ to be significant. For triggered $\mathrm{EMG}$, we consider a threshold value of $5 \mathrm{~mA}$ to be indicative of possible pedicle breach. As with all monitoring, the specific methodology should be clarified with the electrophysiologist prior to each case.

It is the role of the neurosurgeon to establish a clear plan for responding to a positive alarm. Initial investigation should be directed at common sources of false-positive monitoring, including hypotension, hypothermia, and use of halogenated anesthetics. For SSEP and transcranial MEP monitoring, placement of the stimulating and recording electrodes should be verified, because migration or fall-out of leads may cause significant variation in signal amplitude. If this investigation is unrevealing or if true neurological injury is suspected, the surgeon should consider reversing any recent high-risk maneuvers and assessing for improvement in signal amplitude or latency. With triggered EMG, low stimulation thresholds should prompt investigation for medial screw deviation. During open spine surgery, this can be accomplished by manual palpation of the medial pedicle wall. In minimally invasive surgery, assessment may be difficult and screw removal and replacement may be necessary.

Finally, rapid development of new spine surgery techniques, including minimally invasive technologies, can present new challenges for coordination in the operating room. When new techniques are used in the operating room, it is the responsibility of the surgeon to discuss how IONM will be used during the case. This is especially critical during cases in which the anatomy will only be "seen" through the use of fluoroscopy or O-arm. For example, Smith et al. ${ }^{60}$ report a $6.2 \%$ rate of pedicle breach, and a $3.7 \%$ rate of severe breach $(>3 \mathrm{~mm})$ during percutaneous placement of lumbar pedicle screws despite the use of intraoperative fluoroscopic guidance. As such, in cases using minimally invasive techniques or other new technologies, an understanding of how IONM will be implemented throughout the case is critical.

\section{Cost-Effectiveness of IONM and Recommendations for Use}

Despite the marked advancements in neuromonitoring technology, there have been no prospective studies with a high level of evidence performed to validate the efficacy of IONM. Use of IONM is driven largely by surgeon preference and medicolegal concerns. There is a need for prospective studies to establish standardized criteria for use of neuromonitoring; in this report, we present general guidelines for IONM based on the available literature. Use of IONM should largely be dictated by the complexity of the surgical procedure and the assumed risk of new neurological injury. For scoliosis surgery, 
the general consensus is that combined monitoring with SSEPs and transcranial MEPs represents the minimum standard of care. In many cases, triggered EMG may be added for additional nerve root monitoring following instrumentation. Nuwer et al. ${ }^{46}$ compared the results from their 1995 survey of the Scoliosis Research Society to prior data from the same group collected before the widespread use of neuromonitoring. ${ }^{37}$ These authors found a decreased rate of neurological deficits in the newer data and attributed this to the use of SSEP monitoring. While the authors admit that it is impossible to assess the validity of this assumption, they estimate that if SSEP monitoring prevents 1 deficit in every 200 cases, then the cost of preventing 1 new neurological deficit with SSEP monitoring is $\$ 120,000$. This amount is significantly less than even the first-year health care costs of a newly paraplegic patient (http://www.spinalcord.uab.edu; Table 4). ${ }^{14}$

For other forms of spine surgery there are varying levels of evidence. As a specific case example, with intramedullary spinal cord tumor resection the evidence appears to support addition of D-wave monitoring because this correlates most accurately with long-term motor function. ${ }^{30,57}$ However, this form of monitoring is likely not appropriate for other spine cases. Although many would consider IONM to be the standard of care for cervical decompression surgery, not all surgeons agree on this. For instance, in 2012, Traynelis et al. ${ }^{64}$ published a series of 720 consecutive cases of cervical decompression performed without IONM with no new postoperative deficits. Based on CPT codes, the authors estimate the average cost per case (assuming a 4-hour case length) as follows: SSEP, \$941.82; transcranial MEP, \$1114.77; combined SSEP and transcranial MEP, \$1423.27. Based on these estimates, the authors propose that the cost saved by not using combined SSEP and transcranial MEP in these 720 cases is $\$ 1,024,754$. In light of the continually rising costs of health care, this study has raised questions over which cases can be safely performed without neuromonitoring. That said, the use of SSEPs and transcranial MEPs in routine cervical spine decompression and fusion cases is well supported by the scientific literature. ${ }^{35}$ Reports investigating IONM during instrumented anterior cervical surgery suggest a strong role for IONM in alerting the surgical team about neurological changes that may occur during positioning or due to hemodynamic changes during the case. ${ }^{6,32}$ Devlin and colleagues ${ }^{15}$ have suggested that IONM is a useful adjunct during surgery

\section{TABLE 4: Average health care and life expenses attributable to $\mathrm{SCl}^{*}$}

\begin{tabular}{lcc}
\hline $\begin{array}{c}\text { Type of } \\
\text { SCl }\end{array}$ & $\begin{array}{c}\text { First-Yr Costs Following SCl } \\
(\$)\end{array}$ & $\begin{array}{c}\text { Subsequent Annual Costs } \\
(\$)\end{array}$ \\
\hline C1-4 & 775,567 & 138,923 \\
C5-8 & 500,829 & 56,905 \\
paraplegia & 283,388 & 28,837 \\
incomplete & 228,566 & 16,018 \\
\hline
\end{tabular}

* From the National Spinal Cord Injury Statistical Center (http://www. spinalcord.uab.edu). for cervical spondylotic myelopathy. Likewise, Garcia et al. ${ }^{20}$ described the successful use of SSEPs during posterior cervical laminoplasties.

Intraoperative neurophysiological monitoring during lumbar surgery, particularly routine lumbar spine procedures such as uncomplicated decompression and discectomy, is controversial. Although authors of some series have suggested that monitoring in all cases is beneficial, it is not entirely clear whether IONM truly affected the already low neurological complication rates. ${ }^{1,66}$ In revision cases, the higher risk of neurological injury supports the use of neuromonitoring. ${ }^{24}$ Likewise, instrumentation and fusion is associated with a higher risk of nerve root injury, and use of spontaneous EMG or triggered EMG may enhance safety. ${ }^{2,3,7,11}$ In all cases, the experience and skill level of the surgeon should be factored into decision making.

Finally, although use of IONM remains unsupported by prospective studies with a high level of evidence, many surgeons believe it is critical for high-risk cases. The medicolegal implications of neural injury can be significant. In these circumstances, the documentation of the electrophysiology technician can be critical. This documentation provides the surgeon with a timeline of when intraoperative events occurred. Equally, if even more critical, they can demonstrate what steps were taken by the surgeon at the time of an intraoperative event.

To conduct a more complete cost-benefit analysis of IONM, future prospective studies must clarify the rate at which IONM prevents neurological injury. From this information, one could calculate the socioeconomic cost of injury prevented by IONM and compare it to the actual cost of monitoring. By doing so, one could develop a predictive model to assess the financial viability of IONM for various forms of spinal surgery. Given the current state of health care economics, this would be an invaluable tool for assessing which cases warrant use of IONM.

\section{Conclusions}

Intraoperative neurophysiological monitoring is a rapidly evolving field with the potential to greatly improve the safety of spinal surgery. A thorough appreciation of the strengths and weaknesses of each monitoring modality is critical for the optimal use of IONM. Preoperative discussion between the neurosurgeon, anesthesiologist, and electrophysiologist is an essential component of safe IONM usage, and topics should include anesthetic requirements for IONM, alarm criteria to be used, and steps to be taken in response to a positive alarm. Further prospective studies are needed to establish the true efficacy of IONM, but when used properly, IONM represents a powerful tool for improving outcomes in spine surgery.

\section{Disclosure}

Dr. Fessler receives educational grant support for research on spinal techniques from Medtronic, Inc. Dr. Smith was supported by educational grants from the AANS/CNS Spine Section (Apfelbaum Award) and a CNS Spine Fellowship. He has received support for noncompensated educational travel by Medtronic PS Medical. Dr. Koski serves as a consultant to Medtronic and NuVasive.

Author contributions to the study and manuscript prepara- 
tion include the following. Conception and design: all authors. Acquisition of data: Rishi R. Lall. Drafting the article: Smith, Rishi R. Lall, Rohan R. Lall, Hauptman, Ganju. Critically revising the article: all authors. Reviewed submitted version of manuscript: Smith, Rishi R. Lall, Rohan R. Lall, Cybulski. Approved the final version of the manuscript on behalf of all authors: Smith.

\section{References}

1. Akay KM, Onder S: Continuous neural monitoring in lumbar spine surgery: experience with 101 patients. Minim Invasive Neurosurg 45:97-101, 2002

2. Alemo S, Sayadipour A: Role of intraoperative neurophysiologic monitoring in lumbosacral spine fusion and instrumentation: a retrospective study. World Neurosurg 73:72-76, 2010

3. Been HD, Kalkman CJ, Traast HS, Ongerboer de Visser BW: Neurologic injury after insertion of laminar hooks during Cotrel-Dubousset instrumentation. Spine (Phila Pa 1976) 19: 1402-1405, 1994

4. Ben-David B, Haller G, Taylor P: Anterior spinal fusion complicated by paraplegia. A case report of a false-negative somatosensory-evoked potential. Spine (Phila Pa 1976) 12: $536-539,1987$

5. Bose B, Sestokas AK, Schwartz DM: Neurophysiological detection of iatrogenic C-5 nerve deficit during anterior cervical spinal surgery. J Neurosurg Spine 6:381-385, 2007

6. Bose B, Sestokas AK, Schwartz DM: Neurophysiological monitoring of spinal cord function during instrumented anterior cervical fusion. Spine J 4:202-207, 2004

7. Bose B, Wierzbowski LR, Sestokas AK: Neurophysiologic monitoring of spinal nerve root function during instrumented posterior lumbar spine surgery. Spine (Phila Pa 1976) 27: 1444-1450, 2002

8. Calancie B, Harris W, Broton JG, Alexeeva N, Green BA: "Threshold-level" multipulse transcranial electrical stimulation of motor cortex for intraoperative monitoring of spinal motor tracts: description of method and comparison to somatosensory evoked potential monitoring. J Neurosurg 88: 457-470, 1998

9. Calancie B, Lebwohl N, Madsen P, Klose KJ: Intraoperative evoked EMG monitoring in an animal model. A new technique for evaluating pedicle screw placement. Spine (Phila Pa 1976) 17:1229-1235, 1992

10. Calland JF, Turrentine FE, Guerlain S, Bovbjerg V, Poole GR, Lebeau K, et al: The surgical safety checklist: lessons learned during implementation. Am Surg 77:1131-1137, 2011

11. Castellon AT, Meves R, Avanzi O: Intraoperative neurophysiologic spinal cord monitoring in thoracolumbar burst fractures. Spine (Phila Pa 1976) 34:2662-2668, 2009

12. Conley DM, Singer SJ, Edmondson L, Berry WR, Gawande AA: Effective surgical safety checklist implementation. J Am Coll Surg 212:873-879, 2011

13. Deletis V, Sala F: Intraoperative neurophysiological monitoring of the spinal cord during spinal cord and spine surgery: a review focus on the corticospinal tracts. Clin Neurophysiol 119:248-264, 2008

14. DeVivo MJ: Causes and costs of spinal cord injury in the United States. Spinal Cord 35:809-813, 1997

15. Devlin VJ, Anderson PA, Schwartz DM, Vaughan R: Intraoperative neurophysiologic monitoring: focus on cervical myelopathy and related issues. Spine J 6 (6 Suppl):212S-224S, 2006

16. Eager M, Shimer A, Jahangiri FR, Shen F, Arlet V: Intraoperative neurophysiological monitoring (IONM): lessons learned from 32 case events in 2069 spine cases. Am J Electroneurodiagn Technol 51:247-263, 2011

17. Eccher M: Intraoperative neurophysiologic monitoring: are we really that bad? J Clin Neurophysiol 29:157-159, 2012
18. Fan D, Schwartz DM, Vaccaro AR, Hilibrand AS, Albert TJ: Intraoperative neurophysiologic detection of iatrogenic C5 nerve root injury during laminectomy for cervical compression myelopathy. Spine (Phila Pa 1976) 27:2499-2502, 2002

19. Fehlings MG, Brodke DS, Norvell DC, Dettori JR: The evidence for intraoperative neurophysiological monitoring in spine surgery: does it make a difference? Spine (Phila Pa 1976) 35 (9 Suppl):S37-S46, 2010

20. Garcia RM, Qureshi SA, Cassinelli EH, Biro CL, Furey CG, Bohlman HH: Detection of postoperative neurologic deficits using somatosensory-evoked potentials alone during posterior cervical laminoplasty. Spine J 10:890-895, 2010

21. Ginsburg HH, Shetter AG, Raudzens PA: Postoperative paraplegia with preserved intraoperative somatosensory evoked potentials. Case report. J Neurosurg 63:296-300, 1985

22. Gonzalez AA, Jeyanandarajan D, Hansen C, Zada G, Hsieh PC: Intraoperative neurophysiological monitoring during spine surgery: a review. Neurosurg Focus 27(4):E6, 2009

23. Gunnarsson T, Krassioukov AV, Sarjeant R, Fehlings MG: Real-time continuous intraoperative electromyographic and somatosensory evoked potential recordings in spinal surgery: correlation of clinical and electrophysiologic findings in a prospective, consecutive series of 213 cases. Spine (Phila Pa 1976) 29:677-684, 2004

24. Hamilton DK, Smith JS, Sansur CA, Glassman SD, Ames CP, Berven SH, et al: Rates of new neurological deficit associated with spine surgery based on 108,419 procedures: a report of the scoliosis research society morbidity and mortality committee. Spine (Phila Pa 1976) 36:1218-1228, 2011

25. Haynes AB, Weiser TG, Berry WR, Lipsitz SR, Breizat AH, Dellinger EP, et al: A surgical safety checklist to reduce morbidity and mortality in a global population. N Engl J Med 360:491-499, 2009

26. Hilibrand AS, Schwartz DM, Sethuraman V, Vaccaro AR, Albert TJ: Comparison of transcranial electric motor and somatosensory evoked potential monitoring during cervical spine surgery. J Bone Joint Surg Am 86-A:1248-1253, 2004

27. Hsu B, Cree AK, Lagopoulos J, Cummine JL: Transcranial motor-evoked potentials combined with response recording through compound muscle action potential as the sole modality of spinal cord monitoring in spinal deformity surgery. Spine (Phila Pa 1976) 33:1100-1106, 2008

28. Jimenez JC, Sani S, Braverman B, Deutsch H, Ratliff JK: Palsies of the fifth cervical nerve root after cervical decompression: prevention using continuous intraoperative electromyography monitoring. J Neurosurg Spine 3:92-97, 2005

29. Kelleher MO, Tan G, Sarjeant R, Fehlings MG: Predictive value of intraoperative neurophysiological monitoring during cervical spine surgery: a prospective analysis of 1055 consecutive patients. J Neurosurg Spine 8:215-221, 2008

30. Kothbauer KF, Deletis V, Epstein FJ: Motor-evoked potential monitoring for intramedullary spinal cord tumor surgery: correlation of clinical and neurophysiological data in a series of 100 consecutive procedures. Neurosurg Focus 4(5):e1, 1998

31. Langeloo DD, Lelivelt A, Louis Journée H, Slappendel R, de Kleuver M: Transcranial electrical motor-evoked potential monitoring during surgery for spinal deformity: a study of 145 patients. Spine (Phila Pa 1976) 28:1043-1050, 2003

32. Lee JY, Hilibrand AS, Lim MR, Zavatsky J, Zeiller S, Schwartz DM, et al: Characterization of neurophysiologic alerts during anterior cervical spine surgery. Spine (Phila Pa 1976) 31:1916-1922, 2006

33. Lenke LG, Padberg AM, Russo MH, Bridwell KH, Gelb DE: Triggered electromyographic threshold for accuracy of pedicle screw placement. An animal model and clinical correlation. Spine (Phila Pa 1976) 20:1585-1591, 1995

34. Lesser RP, Raudzens P, Lüders H, Nuwer MR, Goldie WD, Morris HH III, et al: Postoperative neurological deficits may occur despite unchanged intraoperative somatosensory evoked potentials. Ann Neurol 19:22-25, 1986 
35. Li F, Gorji R, Allott G, Modes K, Lunn R, Yang ZJ: The usefulness of intraoperative neurophysiological monitoring in cervical spine surgery: a retrospective analysis of 200 consecutive patients. J Neurosurg Anesthesiol 24:185-190, 2012

36. MacDonald DB, Janusz M: An approach to intraoperative neurophysiologic monitoring of thoracoabdominal aneurysm surgery. J Clin Neurophysiol 19:43-54, 2002

37. MacEwen GD, Bunnell WP, Sriram K: Acute neurological complications in the treatment of scoliosis. A report of the Scoliosis Research Society. J Bone Joint Surg Am 57:404408, 1975

38. Magit DP, Hilibrand AS, Kirk J, Rechtine G, Albert TJ, Vaccaro AR, et al: Questionnaire study of neuromonitoring availability and usage for spine surgery. J Spinal Disord Tech 20: 282-289, 2007

39. Malhotra NR, Shaffrey CI: Intraoperative electrophysiological monitoring in spine surgery. Spine (Phila Pa 1976) 35: 2167-2179, 2010

40. May DM, Jones SJ, Crockard HA: Somatosensory evoked potential monitoring in cervical surgery: identification of preand intraoperative risk factors associated with neurological deterioration. J Neurosurg 85:566-573, 1996

41. Merton PA, Morton HB: Stimulation of the cerebral cortex in the intact human subject. Nature 285:227, 1980

42. Minahan RE, Sepkuty JP, Lesser RP, Sponseller PD, Kostuik JP: Anterior spinal cord injury with preserved neurogenic 'motor' evoked potentials. Clin Neurophysiol 112:1442-1450, 2001

43. Nash CL Jr, Lorig RA, Schatzinger LA, Brown RH: Spinal cord monitoring during operative treatment of the spine. Clin Orthop Relat Res (126):100-105, 1977

44. Noonan KJ, Walker T, Feinberg JR, Nagel M, Didelot W, Lindseth R: Factors related to false- versus true-positive neuromonitoring changes in adolescent idiopathic scoliosis surgery. Spine (Phila Pa 1976) 27:825-830, 2002

45. Nuwer MR: A new multicenter survey of neurologic deficits after spinal deformity surgery: are new models of intraoperative neurophysiologic monitoring less accurate? J Clin Neurophysiol 28:602-604, 2011

46. Nuwer MR, Dawson EG, Carlson LG, Kanim LE, Sherman JE: Somatosensory evoked potential spinal cord monitoring reduces neurologic deficits after scoliosis surgery: results of a large multicenter survey. Electroencephalogr Clin Neurophysiol 96:6-11, 1995

47. Obi T, Mochizuki M, Isobe K, Mizoguchi K, Takatsu M, Nishimura Y: Mechanically elicited nerve root discharge: mechanical irritation and waveform. Acta Neurol Scand 100: 185-188, 1999

48. Owen JH: Intraoperative stimulation of the spinal cord for prevention of spinal cord injury. Adv Neurol 63:271-288, 1993

49. Padberg AM, Wilson-Holden TJ, Lenke LG, Bridwell KH: Somatosensory- and motor-evoked potential monitoring without a wake-up test during idiopathic scoliosis surgery. An accepted standard of care. Spine (Phila Pa 1976) 23:1392-1400, 1998

50. Pajewski TN, Arlet V, Phillips LH: Current approach on spinal cord monitoring: the point of view of the neurologist, the anesthesiologist and the spine surgeon. Eur Spine J 16 (Suppl 2):S115-S129, 2007

51. Park P, Wang AC, Sangala JR, Kim SM, Hervey-Jumper S, Than KD, et al: Impact of multimodal intraoperative monitoring during correction of symptomatic cervical or cervicothoracic kyphosis. Clinical article. J Neurosurg Spine 14: 99-105, 2011

52. Parker SL, Amin AG, Farber SH, McGirt MJ, Sciubba DM, Wolinsky JP, et al: Ability of electromyographic monitoring to determine the presence of malpositioned pedicle screws in the lumbosacral spine: analysis of 2450 consecutively placed screws. Clinical article. J Neurosurg Spine 15:130-135, 2011

53. Pelosi L, Jardine A, Webb JK: Neurological complications of anterior spinal surgery for kyphosis with normal somatosen- sory evoked potentials (SEPs). J Neurol Neurosurg Psychiatry 66:662-664, 1999

54. Quraishi NA, Lewis SJ, Kelleher MO, Sarjeant R, Rampersaud YR, Fehlings MG: Intraoperative multimodality monitoring in adult spinal deformity: analysis of a prospective series of one hundred two cases with independent evaluation. Spine (Phila Pa 1976) 34:1504-1512, 2009

55. Raynor BL, Lenke LG, Bridwell KH, Taylor BA, Padberg AM: Correlation between low triggered electromyographic thresholds and lumbar pedicle screw malposition: analysis of 4857 screws. Spine (Phila Pa 1976) 32:2673-2678, 2007

56. Raynor BL, Lenke LG, Kim Y, Hanson DS, Wilson-Holden TJ, Bridwell KH, et al: Can triggered electromyograph thresholds predict safe thoracic pedicle screw placement? Spine (Phila Pa 1976) 27:2030-2035, 2002

57. Sala F, Palandri G, Basso E, Lanteri P, Deletis V, Faccioli F, et al: Motor evoked potential monitoring improves outcome after surgery for intramedullary spinal cord tumors: a historical control study. Neurosurgery 58:1129-1143, 2006

58. Schwartz DM, Auerbach JD, Dormans JP, Flynn J, Drummond DS, Bowe JA, et al: Neurophysiological detection of impending spinal cord injury during scoliosis surgery. J Bone Joint Surg Am 89:2440-2449, 2007

59. Smith PN, Balzer JR, Khan MH, Davis RA, Crammond D, Welch WC, et al: Intraoperative somatosensory evoked potential monitoring during anterior cervical discectomy and fusion in nonmyelopathic patients-a review of 1,039 cases. Spine J 7:83-87, 2007

60. Smith ZA, Sugimoto K, Lawton CD, Fessler RG: Incidence of lumbar spine pedicle breach following percutaneous screw fixation: a radiographic evaluation of 601 screws in 151 patients. J Spinal Disord Tech [epub ahead of print], 2012

61. Taniguchi M, Cedzich C, Schramm J: Modification of cortical stimulation for motor evoked potentials under general anesthesia: technical description. Neurosurgery 32:219-226, 1993

62. Taylor BA, Fennelly ME, Taylor A, Farrell J: Temporal summation - the key to motor evoked potential spinal cord monitoring in humans. J Neurol Neurosurg Psychiatry 56:104106,1993

63. Toleikis JR, Skelly JP, Carlvin AO, Burkus JK: Spinally elicited peripheral nerve responses are sensory rather than motor. Clin Neurophysiol 111:736-742, 2000

64. Traynelis VC, Abode-Iyamah KO, Leick KM, Bender SM, Greenlee JD: Cervical decompression and reconstruction without intraoperative neurophysiological monitoring. Clinical article. J Neurosurg Spine 16:107-113, 2012

65. Ulkatan S, Neuwirth M, Bitan F, Minardi C, Kokoszka A, Deletis V: Monitoring of scoliosis surgery with epidurally recorded motor evoked potentials (D wave) revealed false results. Clin Neurophysiol 117:2093-2101, 2006

66. Weiss DS: Spinal cord and nerve root monitoring during surgical treatment of lumbar stenosis. Clin Orthop Relat Res (384):82-100, 2001

67. Wilson-Holden TJ, Padberg AM, Parkinson JD, Bridwell KH, Lenke LG, Bassett GS: A prospective comparison of neurogenic mixed evoked potential stimulation methods: utility of epidural elicitation during posterior spinal surgery. Spine (Phila Pa 1976) 25:2364-2371, 2000

68. Ziewacz JE, Arriaga AF, Bader AM, Berry WR, Edmondson L, Wong JM, et al: Crisis checklists for the operating room: development and pilot testing. J Am Coll Surg 213:212-217. e210, 2011

Manuscript submitted July 2, 2012.

Accepted September 10, 2012.

Please include this information when citing this paper: DOI: 10.3171/2012.9.FOCUS12235.

Address correspondence to: Zachary A. Smith, M.D., Department of Neurological Surgery, Northwestern Feinberg School of Medicine, 676 North Saint Clair Street, Suite 2210, Chicago, Illinois 60611.email: zsmithmd@gmail.com. 